\title{
Semiautomatic Level-Set Based Segmentation and Stenosis Quantification of the Internal Carotid Artery in 3D CTA Data Sets
}

\author{
Holger Scherl ${ }^{a}$, Joachim Hornegger ${ }^{a}$, Marcus Prümmer ${ }^{a}$, \\ Michael Lell ${ }^{\mathrm{b}}$ \\ a Institute of Pattern Recognition, Department of Computer Sciences, \\ Friedrich-Alexander-Universität Erlangen-Nürnberg, Martensstr. 3, \\ D-91058 Erlangen, Germany \\ ${ }^{\mathrm{b}}$ Institute of Diagnostic Radiology, Friedrich-Alexander-Universität \\ Erlangen-Nürnberg, Maximiliansplatz 1, D-91054 Erlangen, Germany
}

\begin{abstract}
We present a new level-set based method to segment and quantify stenosed internal carotid arteries (ICAs) in 3D contrast-enhanced computed tomography angiography (CTA). Within these data sets it is a difficult task to evaluate the degree of stenoses deterministically even for the experienced physician because the actual vessel lumen is hardly distinguishable from calcified plaque and there is no sharp border between lumen and arterial wall. According to our knowledge no commercially available software package allows the detection of the boundary between lumen and plaque components. Therefore in the clinical environment physicians have to perform the evaluation manually. This approach suffers from both intra- and interobserver variability. The limitation of the manual approach requires the development of a semi-automatic method that is able to achieve deterministic segmentation results of the internal carotid artery via level-set techniques. With the new method different kinds of plaques were almost completely excluded from the segmented regions. For an objective evaluation we also studied the method's performance with four different phantom data sets for which the ground truth of the degree of stenosis was known a priori. Finally, we applied the method to ten ICAs and compared the obtained segmentations with manual measurements of three physicians.
\end{abstract}

Key words: Segmentation; Level-set method; Chan-Vese Model; Computed tomography angiography; Quantification; Stenosis; Internal carotid artery 


\section{Introduction}

One of the major causes of death in western civilization (after coronary artery disease and malignancy) are apoplectic insults, also called apoplexy or stroke. Elder people, in particular, are often affected by disabilities resulting from it. A large percentage of apoplectic insults are caused by stenosed carotid arteries [1]. An atherothrombotic plaque-forming lesion at the bifurcation of the common carotid artery and/or the proximal part of the ICA is often the result of carotid artery atherosclerosis [2]. These plaques cause a gradual, progressive narrowing of the arterial lumen thus making blood flow through the artery progressively more difficult. Lumen means the actual opening of a blood vessel being available to the blood flow. The plaque area often is a thrombus forming site causing a thrombembolia also resulting in an ischemic apoplexy. Treatment of carotid artery stenosis depends on clinical findings related to the degree of the stenosis. In order to determine the degree of a stenosis, there are various ways of imaging. Two-dimensional (2D) intra-arterial digital subtraction angiography (DSA) has historically been used as a standard procedure for visualizing the ICA. However, DSA is an invasive and expensive method with complication rates from $1 \%$ to $4 \%$ [3,4]. Its 2D nature implies limitations for vessels which are located parallel to the viewing direction as well. Magnetic Resonance Angiography (MRA) and CTA are minimally invasive methods that progressively replace DSA in clinical routine. State-of-the-art multislice spiral CT scanners allow for highest spatial resolution at very short scan times (less than 10 seconds). For that reason we used only volume data sets from contrast-enhanced computed tomography for quantification. The disadvantage of CTA-scans is that it is difficult to evaluate the degree of a stenosis in cases of calcified plaques. Previous studies have shown that a significant number of cases had been misclassified [5]. The reasons for these alterations are mainly difficulties arising from manual quantification of CTA-scans. These are in particular: First, to reveal the lumen of the blood vessel on a CT, a contrast agent must be injected. Calcified plaque and blood vessels filled with contrast agent have similar intensity values (in terms of Hounsfield units). Second, for the evaluation, each examiner uses a slightly different intensity window where lumen as well as plaques change their appearance according to the grey scale. Even for the experienced physician it is difficult to distinguish calcified or plaque-attached arterial wall and arterial lumen.

In order to overcome the above mentioned disadvantages, we developed a computerized tool for quantification. So far, no computerized method for quantification of stenosed carotid arteries for CTA data sets is commercially available. We show a novel way of analyzing stenosed blood vessels using a semi-automatic algorithm for quantification, which requires only minimal user interaction. We focus on segmenting the lumen of the vessel only, without including any plaques. Since different sorts of plaque are shown in CTA, it is of 
highest importance for the quantification algorithm to recognize at least two different kinds. While non-calcified plaques have a high penetration for X-rays, the value at a CTA is low thus the non-calcified plaque appears dark. With calcified plaque it is the other way round. The X-rays are more attenuated; therefore the CTA value is high and the plaque looks white and bright. As the appearance of calcified plaques is very similar to that of arterial lumen, it is the most difficult task for any automated method to exclude these plaques from segmentation.

In this article we present a new approach based upon the level-set method [6] that is able to handle these difficulties. We extended the active contour model of Tony F. Chan and Luminita A. Vese [7] to address the vessel/plaque segmentation problem. As we will show the Chan-Vese model is already able to handle data sets that include only non-calcified plaques. But calcified plaques are completely included within the segmentation result. Even worse they are sometimes segmented exclusively. In order to overcome the shortcomings of the Chan-Vese model in the context of the vessel/plaque segmentation problem, we present a modification of that approach and introduce suitable regularization terms. With the proposed extensions it is now possible to use the obtained segmentations for a semi-automatic quantification approach.

This paper is organized as follows. In the next section we review previous approaches to vessel segmentation that are related to stenosis quantification of the ICA. In Section 3 our level-set based method is explained in detail. A major part of this work is devoted to evaluation and validation. Results for phantom data sets and ICAs from different patients are reported in Section 4 and discussed in Section 5.

\section{Previous Work}

Within CTA scans Armin Kanitsar et al. [8] explore the segmentation of peripheral vasculature in large image sequences of the lower extremities. They have to deal with the same problems encountered within this article. Calcified plaques in the vessel walls have to be distinguished from arterial lumen. Their method is strongly dependent on several user-interactions. In [9-12] an automated method for calcification detection in data sets from CTA is presented. With a thresholding approach all connected objects above 220 Hounsfield units are extracted from the scan. In order to distinguish calcifications from noncalcifications a classification of each object is performed based on a number of features, which are computed from the object's size, location, shape characteristics and surrounding structures. In different studies the method detected $75 \%$ to $83.9 \%$ of all calcifications at the expense of some false positives. According to Isgum et al. [10] a main drawback of their approach is that 
in some scans relatively large volumes of contrast agent were misclassified as calcifications. However, in the context of the vessel/plaque segmentation problem such a method could be used to remove calcified plaques from the CTA scan in a pre-processing step. Marcel van Straten et al. address the problem of automatic removal of calcifications in the context of ICA by using a local subtraction method [13]. In their approach an extra CT scan has to be made, prior to contrast injection. The calcifications in both scans are registered rigidly and then subtracted. According to [13], there are some imperfections in the removal of the calcifications caused by patient or calcification motion during data acquisition. However, the method is less subjective and more accurate in comparison to threshold based calcification removal. In [14] a level-set based technique for cerebral vasculature segmentation in CTA is presented that uses a bone masking step based on an additional low-dose mask scan, in order to exclude bones and calcifications. The level set is steered to the vessel boundaries based on an estimation of the background and vessel intensity via an intensity histogram analysis. The achieved quantification results were comparable to the accuracy and reproducibility of two expert observers. The radiation dose for the patient is increased by approximately one third, much more than van Straten et al. [13] needed for the non-enhanced pre-scan.

Some articles have addressed the issue of stenosis grading of ICAs using MRA. In comparison to CTA, a disadvantage of MRA acquisitions is their lower spatial resolution. However, in MRA calcifications are not visualized, thus the vessel/plaque segmentation problem does not occur. Frangi et al. evaluate a 3D model-based approach for the quantification and stenosis grading of ICAs in contrast-enhanced MRA [15] that is comparable to manual quantification. They compute an initial B-Spline surface around the vessel structure from user-indicated points, thus getting a rough approximation of the central axis and fit the surface subsequently to the image data. Also for MRA van Bemmel et al. developed a semi-automatic method for segmentation and stenosis quantification of the ICA [16]. As a pre-processing step, the vessel-enhancing filter from [17] is used. It is constructed to de-enhance non tubular structures. Then, from two user-indicated points, the central vessel axis is determined as the minimum cost path in-between them by considering the reciprocal value of the vessel-enhanced image as cost function. With the central axis serving as initialization, level-set techniques are employed to segment the vessel structure. Level set methods can describe the evolution of implicit active contours driven by external (image based) and internal (contour based) forces. Both the curvature of the contour perpendicular to the vessel axis and the vesselenhanced image including information of the image acquisition are used to define the force terms. The variation in measurements in comparison with manual quantifications was $8.7 \%$ and the results were hardly influenced by parameter variation. The problem of automatic segmentation of vasculature in three-dimensional imagery is also addressed in [18]. Again, level-set methods are used for curve and surface evolution. They use a co-dimension two 
level-set evolution in 3D, i.e., evolving line structures in 3D, in order to evolve tubular structures in a better way compared to curvature-based evolution. Their method was not evaluated quantitatively. Based upon level-set techniques in [19], a method for segmenting multiple regions of simple medical images is shown. The different regions are separated by maximizing the distance between their mean intensity values. The Chan-Vese active contour model without edges [7] is very similar to that kind of approach. Our method is a modification and extension of that model in order to solve the vessel/plaque segmentation problem in data sets from CTA.

According to our knowledge, the only prototypical software tool that faces the problem of performing a quantitative analysis of the carotid arteries in data sets from CTA has been developed by Boskamp et al. [20]. In their work the vessel segmentation is done by a region growing algorithm, that requires the radiologist to manually place several seed points in the vessel of interest. The threshold, which is used by the region growing technique, again has to be adjusted by user interaction. The user has to refine the segmentation by placing several include and exclude markers in order to interactively define regions containing the vessels of interest and regions containing adjacent structures. The obtained segmentation still does not solve the vessel/plaque segmentation problem, because calcified plaques are included in the segmentation. After a skeletonization step the radiologist has to define an analysis path by selecting two points anywhere on the skeleton. Then, another vessel segmentation on a section-by-section basis is computed in several cross-sectional MPRs. Finally, this result is used to perform the quantification of the vessel. According to the NASCET criterion 22 of 29 quantifications are comparable to the reference quantifications by a radiologist using DSA [21]. However, the difference between the radiologist and the semi-automatic approach can be as large as $20 \%$. Concerning the clinical workflow the most repellent disadvantages of this software tool are the numerous interactions required for the radiologist. In contrast, in our method only requires the definition of two points roughly in the middle of the vessel to be analyzed in order to solve the vessel/plaque segmentation problem. One point should be located before the stenosed part and the other one after it. At the same time, these two points could be used to define the vessel analysis path for a fully automatic quantification. However, this problem is not addressed in this paper. The next section gives a detailed description of both the theory of our new approach and the underlying theory. 


\section{Method}

\subsection{Motivation}

Common segmentation approaches like gradient based methods and region growing approaches have difficulties to achieve appropriate segmentations of the arterial lumen especially in the presence of calcifications. Although in CTA the contrast enhanced arterial lumen has a clear contrast differentiation with respect to the background, this is not the case in regions with bones and calcified plaque components. The core regions of calcified plaques have volume densities, that are significantly higher than that of arterial lumen, whereas their border regions have density values like that of arterial lumen. Thus, the volume intensities of these two structures interfere with each other. In Fig. 1a we present $2 \mathrm{D}$ segmentation results achieved with a two dimensional level-set based geodesic active contour model [22]. In an MPR view selected by a radiologist we initialized a circular initial contour (black) in the arterial lumen as indicated by the radiologist. The result of the algorithm (white contour) is shown for different stopping function parameter values. As can be seen, the results are very sensitive to parameter variation. For one configuration the contour stopped as expected but with only small parameter variation the contour did not stop at all or disappeared completely. We also observed, that the results are very sensitive to the initialization of the contour. In Fig.1b results of a simple neighborhood connected region growing algorithm, which includes only voxels having an intensity in a selected interval, are shown for different interval configurations. If the threshold interval is restricted too much, the vessel structures are not completely segmented (left). Using larger threshold intervals border regions of calcified plaques and other neighboring structures are included within the segmentation (middle and right). We also observed that a good threshold interval for one data set is not necessarily a good choice for another one.

Quantifying the degree of stenosis based on such segmentations would be completely useless for the radiologist, because only the contrast enhanced lumen shall influence the quantification result. There are two solutions handling that problem: One can think of a segmentation approach that is able to extract the lumen without including any calcifications by appropriate regularization. The alternative would be a simple segmentation approach, that includes the calcifications in a first step, and then tries to remove them in a second step. As a reliable removal of the calcifications is very difficult to achieve without using a non-enhanced pre-scan, it is very likely that several additional user interactions are required in these approaches. That's why we decided to develop a segmentation algorithm that tries to exclude background regions and calcifications using certain regularization during the segmentation step. 

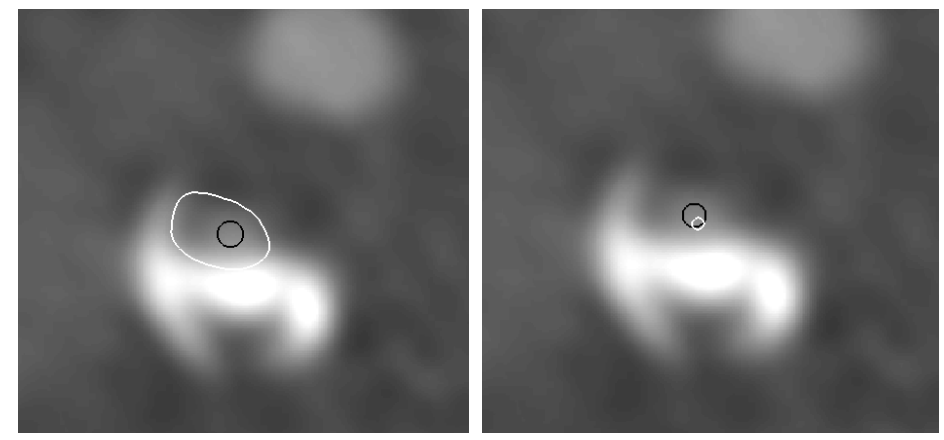

(a)
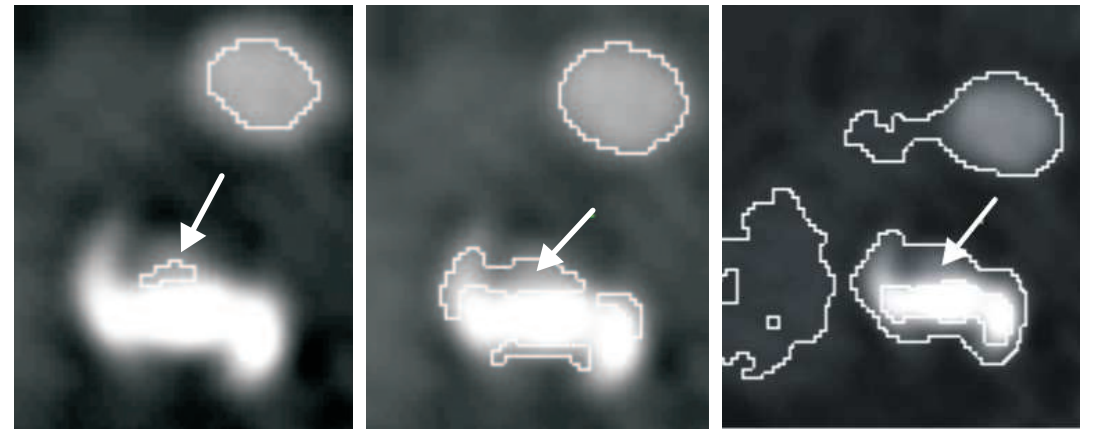

(b)

Fig. 1. Segmentation results with two well known methods are shown in an axial MPR view, that was selected by a radiologist at the stenosed vessel part. In (a) results of the geodesic active contour framework are shown. The initial contour (black) is initialized inside the arterial lumen of the vessel as indicated by the radiologist. The white contour represents the segmentation result after 1000 iterations (left) and 122 iterations (right) with slightly different parameter settings. In (b) the segmentation results (white contour) of a threshold based region growing approach are shown. From left to right the threshold interval is successively increased.

\subsection{Active Contours without Edges}

The active contour model without edges of Chan and Vese [7] is able to detect objects that are not necessarily defined by strong gradient magnitude. It is based on techniques of curve evolution and level sets. In the level-set framework introduced by S. Osher and J. Sethian [6] an implicit representation of the contour is used. A very important property of this method is that two- and three-dimensional problems can be treated in one and the same formalism. Furthermore it allows automatic topology changes, such as merging and breaking and also has the ability to represent cusps and corners of the interface. Within the variational active contour framework, a volume $u_{0}$ is considered as a real-valued function $u_{0}: \Omega \subset \mathbb{R}^{3} \rightarrow \mathbb{R}$, where $\Omega$ is a non empty open and bounded set with Lipschitz boundary $\partial \Omega$ (usually $\Omega$ is a cube in $\left.\mathbb{R}^{3}\right)$. Throughout, $\vec{x}=(x, y, z)$ denotes a voxel position in $\Omega,|\cdot|$ stands for the Euclidean norm, " $\nabla$ " denotes the gradient and "div" the divergence operator. 
The level-set method implicitly represents a curve $C_{0}$ within a higher dimensional function as the boundary of an open set $\omega$. We represent the curve $C_{0}$ as the zero level-set of a scalar Lipschitz function $\phi: \mathbb{R}^{3} \rightarrow \mathbb{R}$, such that

$$
\left\{\begin{array}{l}
\phi(\vec{x})>0 \text { in } \omega \\
\phi(\vec{x})<0 \text { in } \Omega \backslash \omega \\
\phi(\vec{x})=0 \text { on } \partial \omega .
\end{array}\right.
$$

For the implicit representation of the curve $C_{0}$, one can define the function $\phi$ using the signed Euclidean distance function [22]. For more details on the level-set method and its applications we refer the reader to [23].

The active contour model without edges looks for a particular partition of the given volume into two regions, one representing the objects to be detected, and the second one representing the background. The active contour is given as the boundary between the two regions and does not necessarily have to be defined by gradient. This is achieved by the minimization of an energy based functional using the intensity statistics (mean value) of the volume data set inside and outside the evolving implicit curve. The following variational integral is considered:

$$
\begin{aligned}
F_{1}\left(\phi(\vec{x}), c_{1}, c_{2}\right) & =\alpha \int_{\Omega}|\nabla H(\phi(\vec{x}))| d \vec{x}+\gamma_{1} \int_{\Omega}\left|u_{0}(\vec{x})-c_{1}\right|^{2} H(\phi(\vec{x})) d \vec{x} \\
& +\gamma_{2} \int_{\Omega}\left|u_{0}(\vec{x})-c_{2}\right|^{2}[1-H(\phi(\vec{x}))] d \vec{x},
\end{aligned}
$$

where $c_{1}$ and $c_{2}$ are the mean values of the image $u_{0}$ inside and outside the curve defined as the zero level-set of $\phi$ and $H(z)$ denotes the Heaviside function. The weight factors $\alpha, \gamma_{1}$ and $\gamma_{2}$ are the regularizing parameters. Intuitively, the first term of the energy functional punishes high curvatures of the contour. The next two terms increase the energy proportional to the absolute differences of the intensity values and the mean intensity value of the corresponding region (object/background). This model performs as active contours, looking for a 2-phase segmentation of the volume.

Considering $H_{\epsilon}$ and $\delta_{\epsilon}$ any $C^{1}$ approximations and regularizations of the Heaviside function $H$ and Delta function $\delta_{0}$, as $\epsilon \rightarrow 0$ and with $H_{\epsilon}^{\prime}=\delta_{\epsilon}$, and minimizing the energy with respect to $\phi$, we obtain the gradient descent flow: 

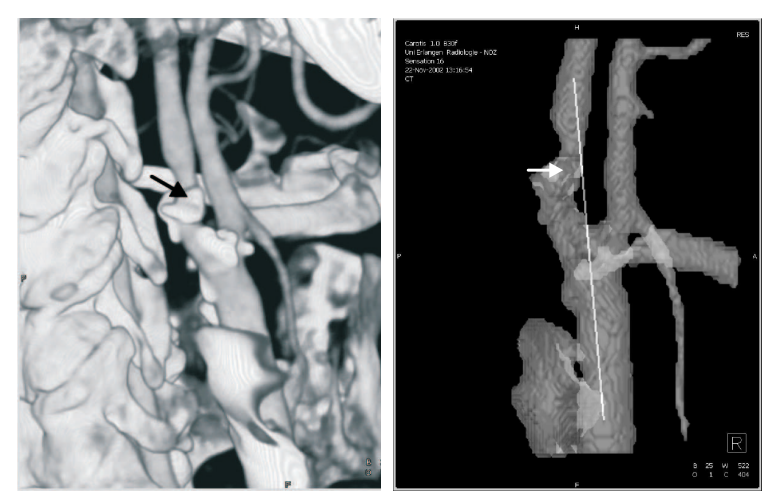

(a)
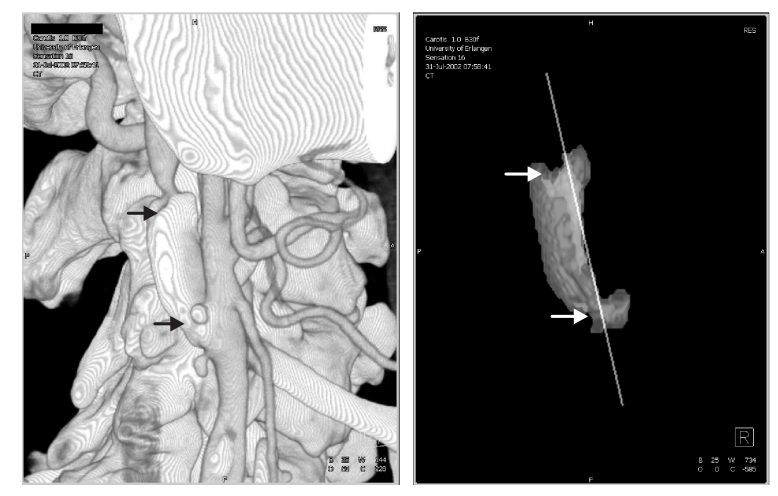

(b)
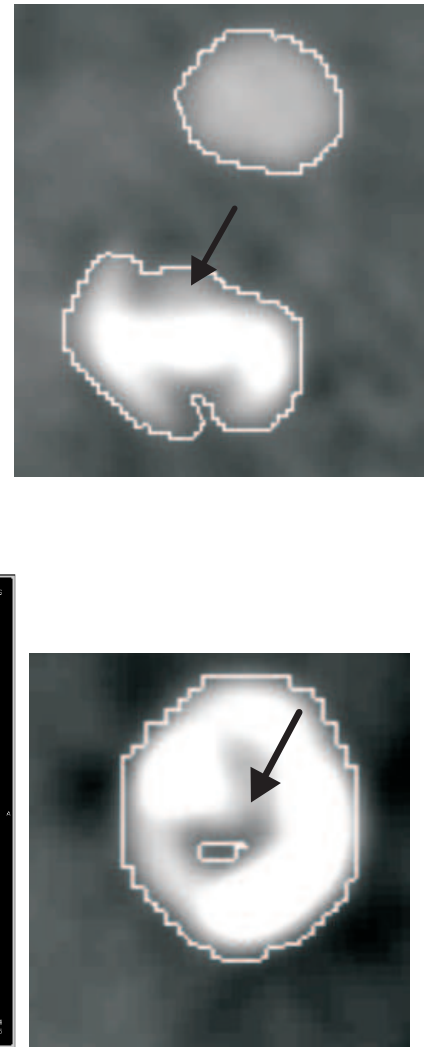

\section{(1)}

Fig. 2. Two data sets with high calcium burden are shown $(\mathrm{a}, \mathrm{b})$. We give in each sub-figure (left) a VRT (Volume Rendering Technique) and the obtained segmentation results of the Chan-Vese model as a 3D surface (middle). An MPR (right) at the stenosed part is also given. The segmentation result is visualized by the white contour. In each figure the location of stenosis is indicated by arrows.

$$
\begin{aligned}
\frac{\partial \phi(\vec{x})}{\partial t}=\delta_{\epsilon}(\phi(\vec{x}))( & \alpha \operatorname{div}\left(\frac{\nabla \phi(\vec{x})}{|\nabla \phi(\vec{x})|}\right) \\
& \left.-\gamma_{1}\left(u_{0}(\vec{x})-c_{1}\right)^{2}+\gamma_{2}\left(u_{0}(\vec{x})-c_{2}\right)^{2}\right) .
\end{aligned}
$$

We observed that the Chan-Vese model performed very well for the segmentation of arterial lumen in data sets without any calcified plaque components [24]. However, in data sets with high calcium burden, calcified plaques were included completely in the segmented regions. This can be seen in Figure 2 which shows segmentation results for ICAs with high calcium burden. Thus, the segmentation with the Chan-Vese method is not feasible for real clinical quantification unless the problem of the inclusion of high intensity anatomical structures like bones and calcifications into the segmented regions is solved. However, in their core regions the intensities of these structures are significantly higher than that of the arterial lumen. We take advantage of this observation to derive a new model that overcomes the above mentioned problems. It will be described in detail in the next section. 


\subsection{The Proposed Model: Extension to Vessel Segmentation without Plaque}

The basic idea of our new model is to handle the background region differently in order to be able to include plaques and bones of high intensity into the background region. Now, two mean intensity values, $c_{2}$ and $c_{3}$, are calculated for its representation while the arterial lumen, which is to be segmented, is still represented by the mean intensity $c_{1}$. The intensity $c_{2}$ is calculated from all background voxels that are smaller than $c_{1}$ and the intensity $c_{3}$ is calculated from all background voxels that are greater or equal to $c_{1}$. With these modifications compared to [7], we are able to include both low and high intensities into the background regions. Now, we formulate the energy to be minimized as

$$
\begin{gathered}
F_{2}\left(\phi(\vec{x}), c_{1}, c_{2}, c_{3}\right)=\alpha \int_{\Omega}|\nabla H(\phi(\vec{x}))| d \vec{x}+\gamma_{1} \int_{\Omega}\left|u_{0}(\vec{x})-c_{1}\right|^{2} H(\phi(\vec{x})) d \vec{x} \\
+\gamma_{2} \int_{\Omega} \min \left(\left|u_{0}(\vec{x})-c_{2}\right|^{2},\left|u_{0}(\vec{x})-c_{3}\right|^{2}\right)[1-H(\phi(\vec{x}))] d \vec{x} .
\end{gathered}
$$

Here, "min(.,.)" denotes the minimum of its arguments. But within this formulation it appears that it is not possible to control to which mean intensity $c_{1}$ the segmented region is going to be attracted. Sometimes, only the borders of the arterial lumen were segmented and the inner part of the lumen was considered as high intensity background. For that reason, we decided to include an additional regularizing term:

$$
F_{3}\left(\phi(\vec{x}), c_{1}, c_{2}, c_{3}\right)=F_{2}\left(\phi(\vec{x}), c_{1}, c_{2}, c_{3}\right)+\lambda \int_{\Omega}\left|u_{0}(\vec{x})-\mathcal{E}\right|^{2} H(\phi(\vec{x})) d \vec{x}
$$

With this extension to the Chan-Vese functional, we penalize all parts of the segmented region that are different to a stable and representative intensity estimate $\mathcal{E}$ of arterial lumen, where $\lambda$ is a constant parameter. Additionally, we forced the mean intensity $c_{3}$ to be at least $\mathcal{E}+\Delta$ in data sets that have no voxels with a higher intensity than the arterial lumen. With the intensity values being normalized to an interval of [0..1], we figured out that a value of $\Delta=0.1$ is a good choice.

The energy $F_{3}$ often leads to excellent results for data sets that included mainly non-calcified plaques with low intensity. The core regions of calcified plaques were correctly excluded from the segmentation. Still, the segmentation result in regions with calcified high intensity plaques did not prove to be satisfactory. The main problem is that all kinds of calcified plaques have an intensity profile at their borders that equals that of the arterial lumen. Thereby, the segmented regions also included the border voxels of calcified plaques, as shown in Fig. 3a. 


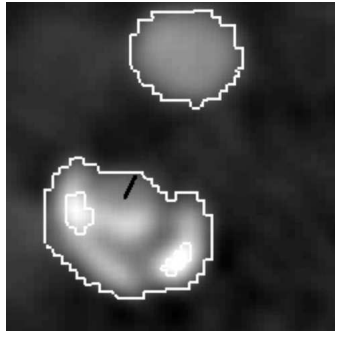

(a)

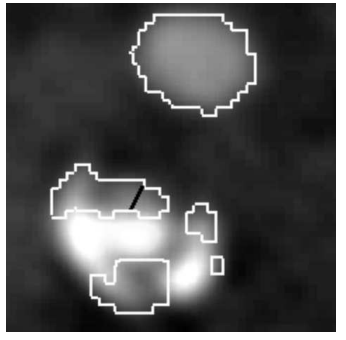

(b)

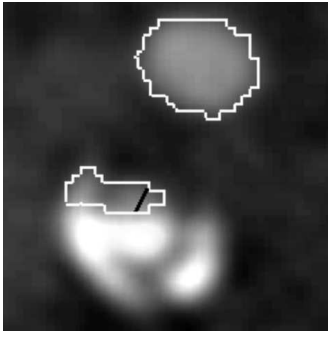

(c)

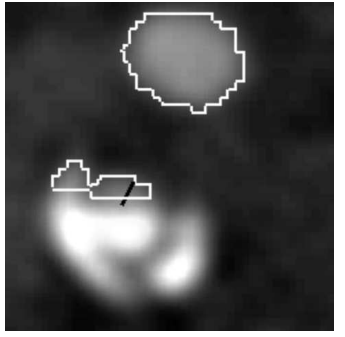

(d)

Fig. 3. The segmentation (white contour) at the border of calcified plaques is shown without regularization (a) and with regularization (b-d). The values of $\mu$ were chosen as $\mu=\{0,10,20,30\}$ in that order. In each sub-figure a measurement from a physician is indicated by a black line for comparison.

In order to reduce the inclusion of these border voxels to the segmented regions, we added an additional regularization term:

$$
\begin{aligned}
F_{4}\left(\phi(\vec{x}), c_{1}, c_{2}, c_{3}\right)= & F_{3}\left(\phi(\vec{x}), c_{1}, c_{2}, c_{3}\right) \\
& +\mu \int_{\Omega} \max _{\vec{x} \in \mathcal{N}_{P}}\left(u_{0}(\vec{x})-\mathcal{E}, 0\right) H(\phi(\vec{x})) d \vec{x} .
\end{aligned}
$$

The basic idea is to force voxels almost having the same intensity like the arterial lumen to be excluded from the segmentation when they are located in the vicinity of calcified plaques. Therefore, we considered at each voxel its cubic neighborhood $\mathcal{N}_{P}$ within a radius of two voxels to penalize voxels that have intensities higher than that of the estimate $\mathcal{E}$ of arterial lumen in their neighborhood. The constant parameter $\mu$ determines the potential of regularization. Fig. 3 (b-d) shows the regularizing effect for different values of $\mu$. Unfortunately, the introduction of the various regularization terms increased the dimension of the parameter space, thus making it more difficult to select a suitable parameter configuration. However, in Section 4.2 we will arrive at a satisfying parameter setting for the problem of vessel segmentation in CTA.

Using the Euler-Lagrange variational principle and considering again $H_{\epsilon}$ and $\delta_{\epsilon}$ any $C^{1}$ approximations and regularizations of the Heaviside function $H$ and Delta function $\delta_{0}$, as $\epsilon \rightarrow 0$ and with $H_{\epsilon}^{\prime}=\delta_{\epsilon}$, and minimizing the energy with respect to $\phi$, we obtain the gradient descent flow:

$$
\begin{aligned}
\frac{\partial \phi(\vec{x})}{\partial t}=\delta_{\epsilon}(\phi(\vec{x}))( & \alpha \operatorname{div}\left(\frac{\nabla \phi(\vec{x})}{|\nabla \phi(\vec{x})|}\right)-\gamma_{1}\left(u_{0}(\vec{x})-c_{1}\right)^{2} \\
& +\gamma_{2} \min \left(\left(u_{0}(\vec{x})-c_{2}\right)^{2},\left(u_{0}(\vec{x})-c_{3}\right)^{2}\right) \\
& \left.-\lambda\left(u_{0}(\vec{x})-\mathcal{E}\right)^{2}-\mu \max _{\vec{x} \in \mathcal{N}_{P}}\left(u_{0}(\vec{x})-\mathcal{E}, 0\right)\right) .
\end{aligned}
$$




\subsection{Medical Work Flow and Required Interactions}

In order to evaluate the degree of stenosis manually the radiologist has to carry out several steps. He has to navigate through a three-dimensional data set and select at least the location of stenosis and a healthy location within the internal carotid artery. Multi planar reconstructions (MPR) of the volumes are used for this task. The quantification itself requires the measurement of the minimal diameters of arterial lumen at these locations. The radiologist is able to use his experience to distinguish the lumen of vessels from different kinds of plaques by following the arteries and by anticipating their distribution. While an automated method has to do without such knowledge, our approach requires only two additional user-defined points for its segmentation task, one being located before the stenosed part of the vessel and the other one after it. Special care has to be taken in order to select points that are approximately in the middle of the lumen within the axial view. At the same time, these two points could be used to define the vessel analysis path for a fully automatic quantification procedure. However, this is not addressed in this paper.

\subsection{Implementation}

In order to discretize Equation (7), we used a finite differences approach. Additionally, for the approximation of the mean curvature we used the AOS scheme described in [22]. Therefore, we were able to use a rather large time step size of $\Delta t=1.0$. Because of numerical reasons, we re-distanced the level-set function at least every 5 iterations. We used the fast re-distancing method introduced in [25]. A narrow band that only updates the points within 3 voxels on either side of the zero level-set was used to speed up the evolution [26]. We incorporated the update of the narrow band within the re-distancing step. For a faster convergence, we initialized the level-set function with the result of a simple threshold-based region growing. We asked the radiologist to select two points $\mathcal{E}_{1}$ and $\mathcal{E}_{2}$ of the arterial lumen, one being located before the stenosed part of the vessel and the other one after it. In order to select appropriate interval limits, we evaluated results of the region growing applied to ten different data sets with varying interval configurations. Then, the intensity interval of the region growing algorithm was chosen to be

$$
\left[\mathcal{E}_{\text {min }}^{M}, \mathcal{E}_{\text {max }}^{M}\right]=\left[\min \left(\mathcal{E}_{1}^{M}, \mathcal{E}_{2}^{M}\right)-0.04, \max \left(\mathcal{E}_{1}^{M}, \mathcal{E}_{2}^{M}\right)+0.04\right]
$$

where $\mathcal{E}_{1,2}^{M}$ denotes the mean intensity in the neighborhood of voxel $\mathcal{E}_{1,2}$, respectively. The mean intensity of all voxels that were included within the initial segmentation is used as the intensity estimate $\mathcal{E}$ of arterial lumen. In order to further reduce the computational burden we also restricted our cal- 
culations to a certain region of interest (ROI). We set the size of the ROI to $101^{3}$ voxels. The midpoint of the ROI was easily selected at the point of gravity of the given points $\mathcal{E}_{1,2}$. On an Intel Pentium 4 machine running at $3 \mathrm{GHz}$ with 2 GBs of memory, the computation time of our method is between four and five minutes depending on the data set. However, we did not spend much time in optimizing our method regarding to execution speed on a particular machine.

\section{Experimental Evaluation}

\subsection{Method of Evaluation}

It was a challenging task to compare the results of the proposed method with the radiologist's measurements. Because of the manual evaluation procedure, no deterministic results were obtainable from the experts. Moreover, we have not yet implemented an automatic quantification procedure. For an objective comparison of the method's performance we used 4 phantom data sets in which the degree of the stenosis was known a priori. We also applied our method to CTA data sets from ten different patients. Three radiologists evaluated the data sets of each patient twice, within an approximately two-week interval to ensure blinded inter- and intra-observer variability. For each ICA the observers modified orientations and positions of a multi-planar reformation (MPR) with the clinical software "InSpace3D" from HipGraphics. We integrated the measurement application itself into the used software tool as a plugin. In the resulting two-dimensional reformations of the specified planes, the observers evaluated the minimal diameter of the arterial lumen at the stenosis (minimal residual lumen $d$ ) and at a reference part (distal ICA lumen diameter $D$ ) by selecting two points. We determined the measured quantities by the length of the lines in-between these points and compared the result of the algorithm directly with all measurements from the observers in the corresponding planes. Therefore, we reformated for each observer the segmentation result of the method by trilinear interpolation into the corresponding plane of an observer's measurement and computed the measured length of the algorithm in this plane by the previously defined points from the corresponding observer.

In a first step, we determined the point of gravity for the two points. Then, we searched for the contour positions of the segmentation along this line in both directions. If the point of gravity was not included in the segmented region, we selected the point on that line being part of the segmented region with minimal distance to that point as the first contour point and measured the length until we reached the next intersection with the contour in the same 
direction. With this approach, we obtained for each observation $O$ a corresponding measurement quantity $M$ for the algorithm and we supposed the mean value of differences between $M$ and $O$, that is $(M-O)_{\text {mean }}$, to be an approximation of error in the algorithm's result. It has to be mentioned that it was not possible to evaluate the result of the algorithm for a specific observer when the corresponding line did not intersect the segmented region at all. Finally, we computed the degree of the stenosis for both the measurements of the radiologist and the corresponding measurements of our method by applying the NASCET criterion [27]:

$$
\text { degree of stenosis }(\%)=\frac{D-d}{D} \times 100 \text {. }
$$

\subsection{Regularization Optimization}

The method's potential of regularization is influenced by various parameters. It is very difficult to find suitable parameter settings because the parameter space has five dimensions. Therefore, testing all combinations of parameter values was impossible in a reasonable time. As a starting point for the evaluation, we chose a suitable configuration of parameters by inspection and tested the influence of parameter variation only within a certain range around the chosen configuration. While the parameter $\lambda$ only translated suitable ranges of other parameters, we kept it fixed to a value of $\lambda=100$ throughout the evaluation. We also restricted the $\gamma$-parameters to $\gamma=\gamma_{1}=\gamma_{2}$. Thereby, the parameter space was limited enough to test the influence of parameter variations in a reasonable time. The first objective was to find suitable values for the parameters $\alpha$ and $\gamma$. Both parameters were varied independently with $\gamma \in\{200,220,240,260,280,300,320,340,360,380,400\}$ and $\alpha \in\{0.25,0.50,0.75\}$ by keeping the parameter $\mu$ fixed at $\mu=10$. We chose randomly two ICAs with calcified plaques and two ICAs with non-calcified plaques for the evaluation.

In Fig. 4a, the mean error of the measurements is shown as a function of $\gamma$ for different $\alpha$-values. The symbols indicate that the algorithm produced measurements for all observers of the corresponding parameter configuration. It can be seen that for increasing values of $\gamma$, the segmentation results differed more from that of the observers. For each $\alpha$ a point was reached at some value of $\gamma$ where the minimal variance terms outweighed the influence of the mean curvature regularization. For $\alpha=0.75$ the algorithm did not produce results for all observers $(\gamma=200,220,240)$. Therefore, we considered the parameter configuration with $\alpha=0.5$ and $\gamma=220$ to be optimal. With this configuration, the algorithm was not only able to produce results for all observers but also the mean errors according to the observers were lowest. Furthermore, the variation in the parameters of $\alpha$ or $\gamma$ was minimal. 

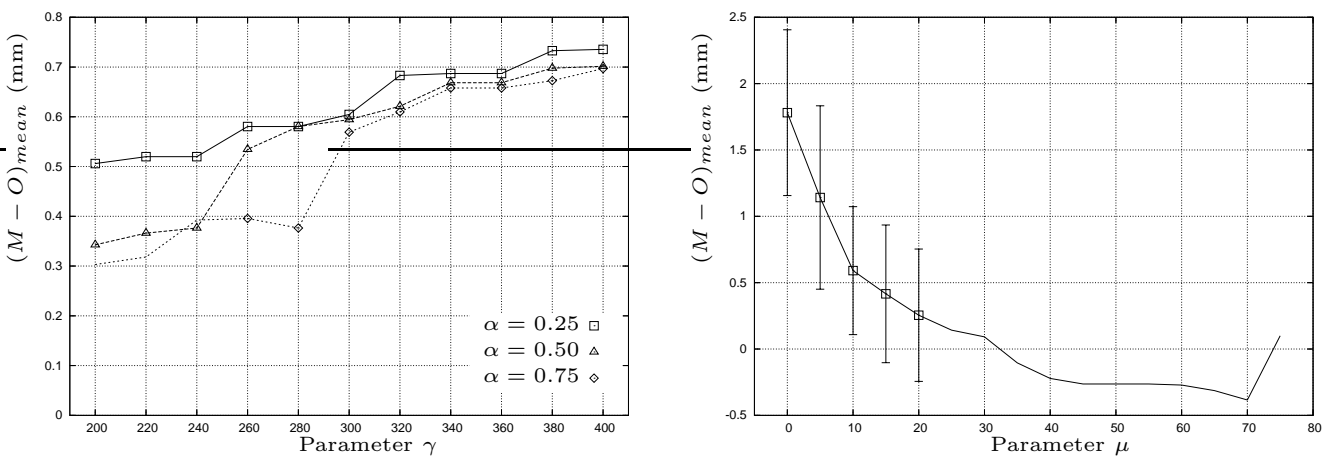

Fig. 4. Average difference between the minimal diameter of the arterial lumen at the stenosis as determined by the method $(\mathrm{M})$ and by the corresponding observer $(\mathrm{O})$ as a function of $\gamma(\mathrm{a})$ and as a function of $\mu(\mathrm{b})$.

In order to test the influence of the parameter $\mu$ on the segmentation results, we additionally evaluated its influence for $\mu \in\{0,10,20,30,40,50,60,70\}$. The other parameters were fixed to the supposed optimal values mentioned above. Fig. $4 \mathrm{~b}$ visualizes the results obtained by the evaluation of two ICAs with calcified plaques. It can be seen that the regularization constraint actually improved the resulting segmentation according to the observers. However, for values of $\mu \geq 30$ the algorithm was not able to produce measurements for all observers any more. We supposed a value of $\mu=10$ to be a good choice, because it is very likely that the algorithm is able to produce results while being regularized enough.

\subsection{Results}

As mentioned in the previous section, one satisfying parameter configuration was $\alpha=0.5, \gamma=220, \lambda=100$ and $\mu=10$. We used this configuration of parameters to compute results with the algorithm both for four phantoms and for ten different ICAs.

\subsubsection{Experiments with Phantoms}

For an objective comparison of the method's performance we applied our algorithm to four different phantom data sets with an a priori known degree of stenosis. The dimensions of the phantoms (diameter of the lumen at reference part and at part of stenosis) are $6.0 / 4.0 \mathrm{~mm}$ (a), 4.0/3.0 mm (b), $6.0 / 2.0 \mathrm{~mm}$ (c) and 4.0/1.5 $\mathrm{mm}(\mathrm{d})$. The data sets were recorded at a 16row CT system. The average density was $200 \mathrm{HU}$ for the lumen and $900 \mathrm{HU}$ for the phantom. Fig. 5 gives a visualization of the obtained segmentations for two phantoms. Table 1 lists the obtained results. The average error was $4.3 \%$ and the maximum error was $9.8 \%$. 


\begin{tabular}{|c||c|c|c|c|}
\hline Phantom & Real [\%] & Measured [\%] & Error [\%] & Diameter of Stenosis [mm] \\
\hline a & 33,3 & 33,4 & 0,1 & 4.0 \\
b & 25,0 & 26,7 & 1,7 & 3.0 \\
c & 66,7 & 72,1 & 5,4 & 2.0 \\
d & 62,5 & 72,3 & 9,8 & 1.5 \\
\hline
\end{tabular}

Table 1

The degree of stenosis of different phantom data sets as it is physically given and as it is measured by the algorithm. The error and the physically given diameter of stenosis is also given.

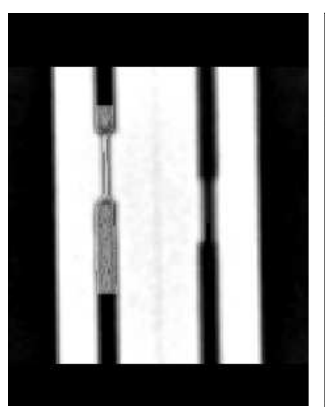

(a)

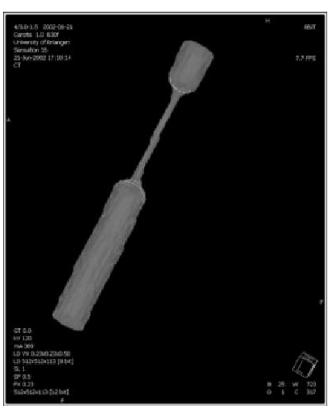

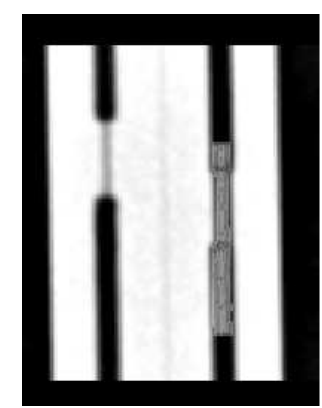

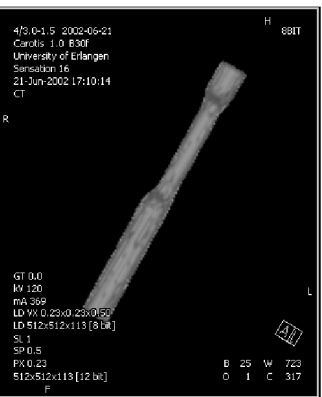

(b)

Fig. 5. The segmentations of two phantom data sets are shown, each sub-figure gives an MPR view and a 3D surface of the segmented region, which is indicated as a grey boundary or surface.

\subsubsection{Experiments with Real Data Sets}

For further evaluation CTA data sets of ten patients with a symptomatic stenosis of the carotid artery were considered. We recorded the data sets at a 16-row CT system. In order to get a representative collection of ICAs, we included patients with non-calcified and calcified plaques and/or both types. In Fig. 6, we give a visualization of the obtained results for each ICA. We show on the left side in each sub-figure a VRT view (Volume Rendering Technique) and on the right side the three-dimensional surface of the resulting segmentation. The ICAs (a) and (g) had a rather poor contrast agent timing which caused the internal jagular vein to appear contrast-enhanced, too. In (b), (d), (f) and (h), data sets with high calcium burden are shown.

Figures 7 and 8 show in each row from left to right four MPR views, which were selected by the observers in order to measure the minimal diameter for the corresponding ICA. The first two MPRs in each row show two measurements of the observers that were in good agreement with the computed segmentation result. In contrast, the last two MPRs are measurements of the observers that did not compare to the computed segmentation result as well. In each view, the observer's measurement is indicated by a black line and the reformated result of our method is indicated by white contours. With our evaluation approach 


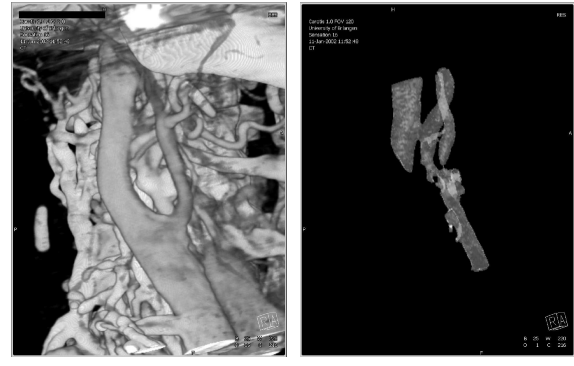

(a)

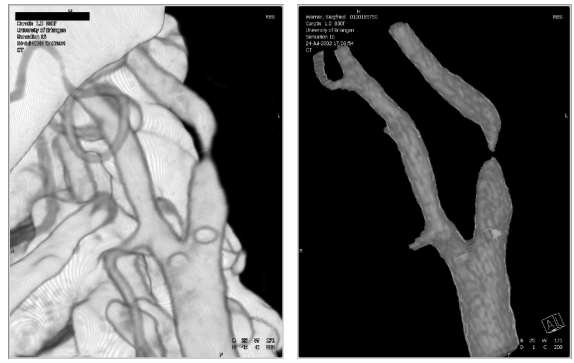

(c)

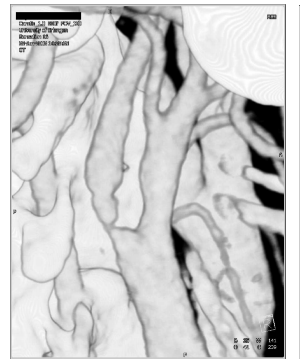

(e)

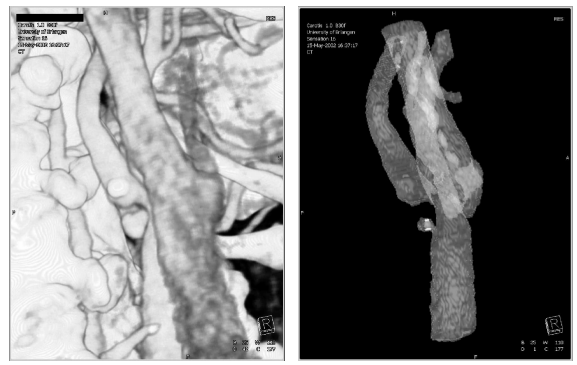

(g)
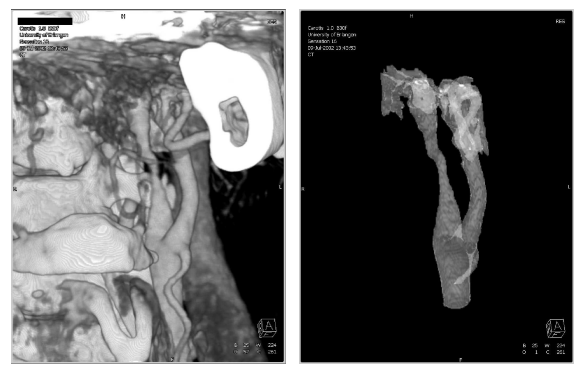

(i)

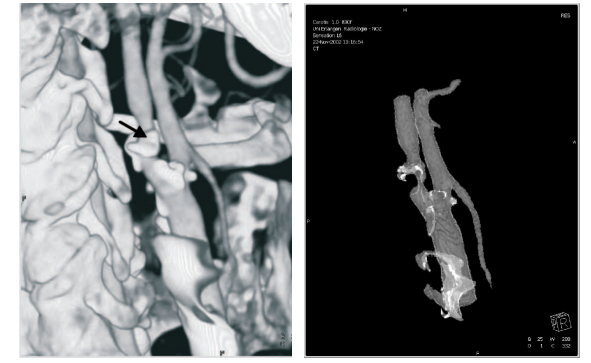

(b)

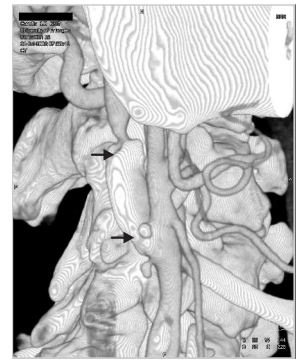

(d)

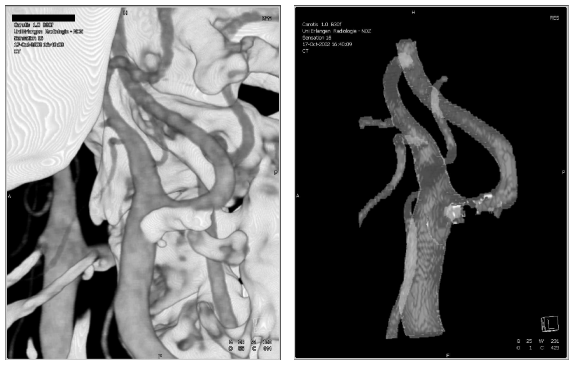

(f)

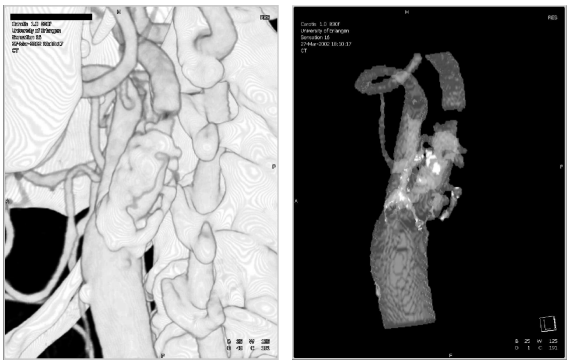

(h)

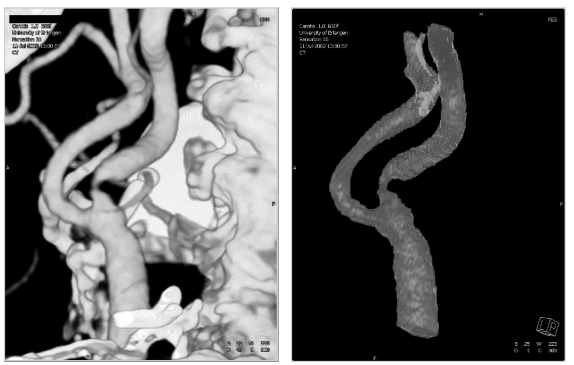

(j)

Fig. 6. Each sub-figure shows a VRT of an ICA (left) and the accompanying segmentation as a three-dimensional surface (right). 
(a)
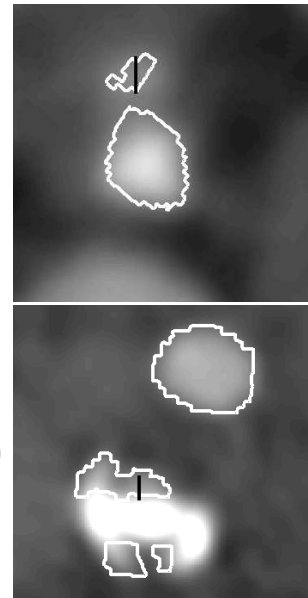

(b)

(c)

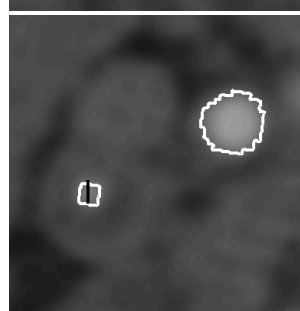

(d)

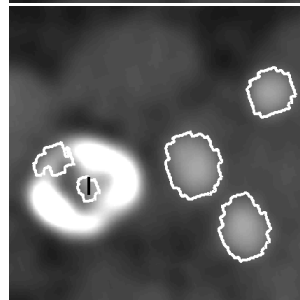

(e)

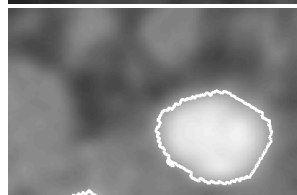

(1)
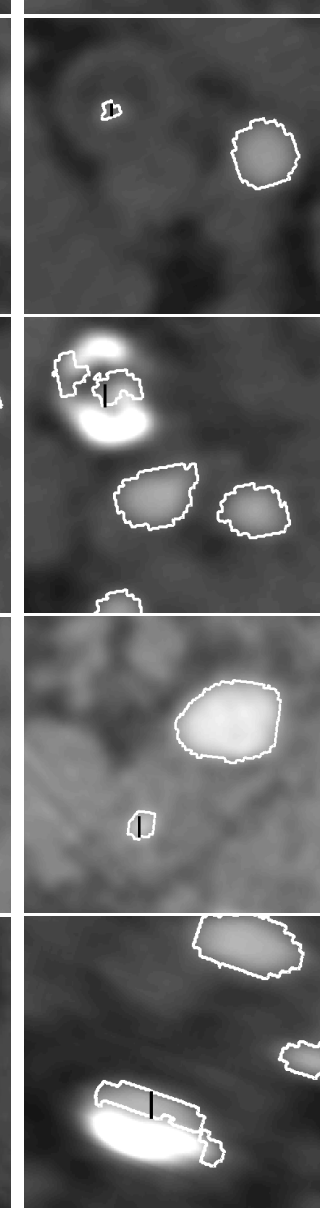

(f)

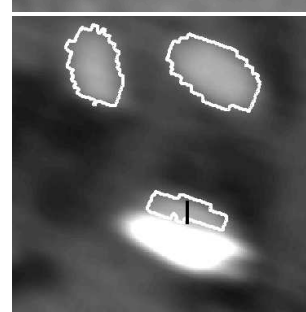

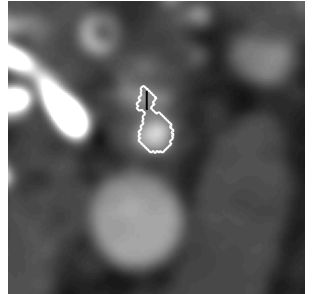
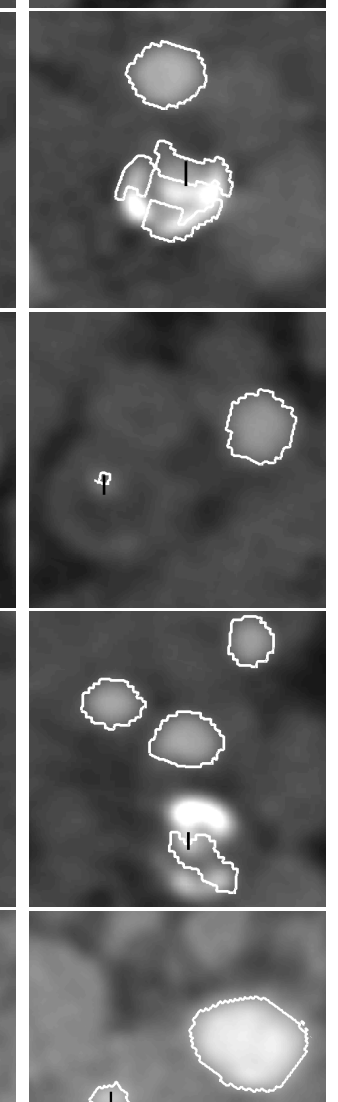

4)
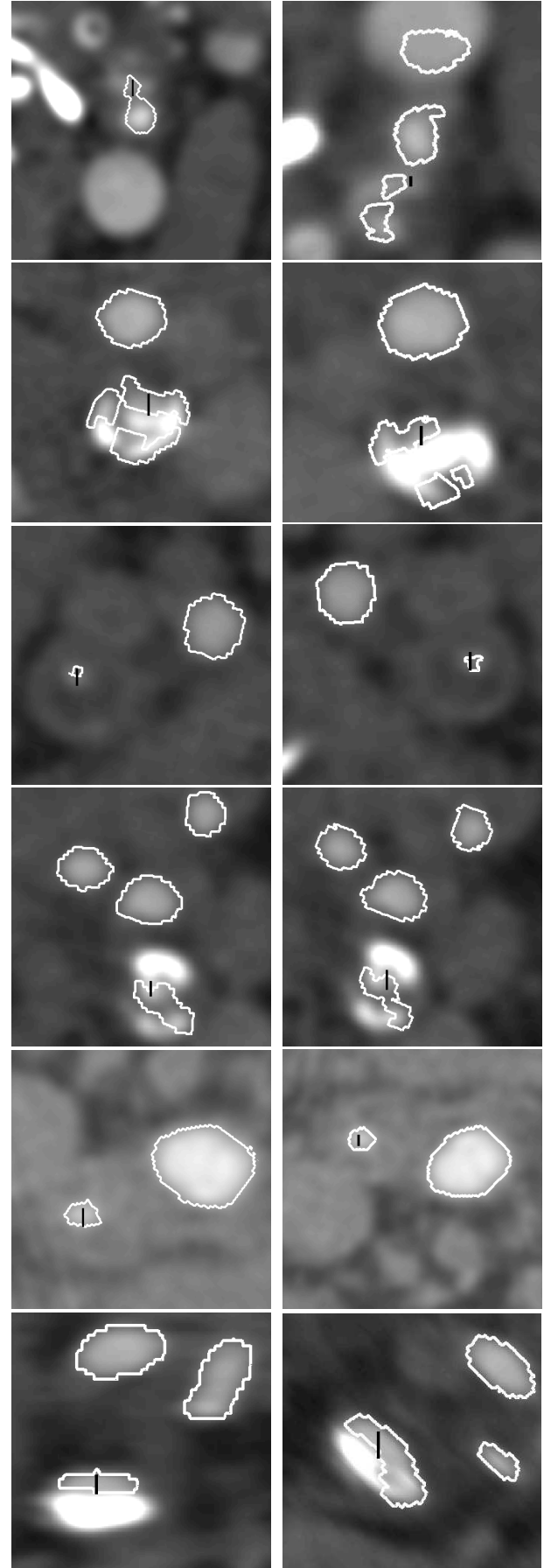

Fig. 7. For ICAs (a) to (f) we show for some measurements the corresponding MPRs at the location of the stenosis. We give in each row for a specific patient MPR views that were used by the observers for their evaluations. We tried to include from left to right at least two good results and two results that did not perform as well. In each picture we indicate the measurement of a radiologist (black line) and the segmentation of our method (white contours). 
$(\mathrm{g})$

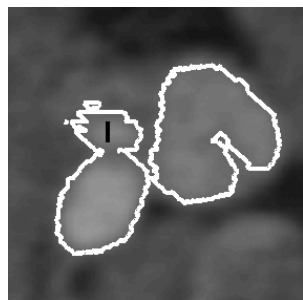

(1)

(h)

(i)

(j)
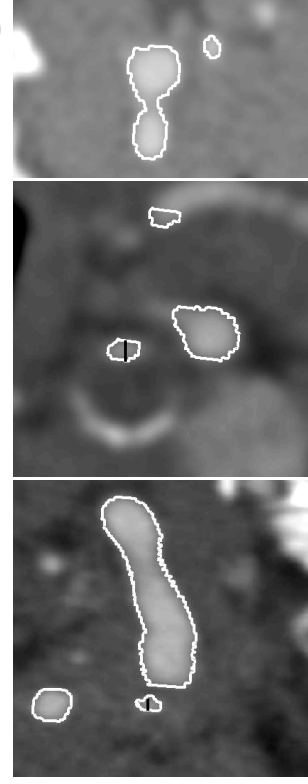
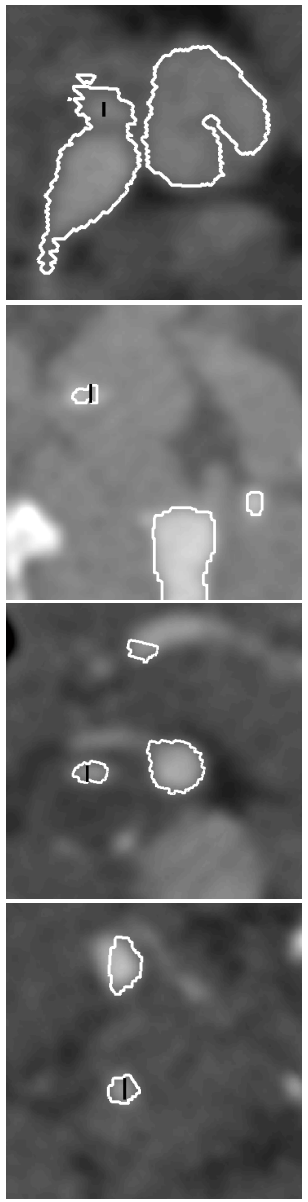
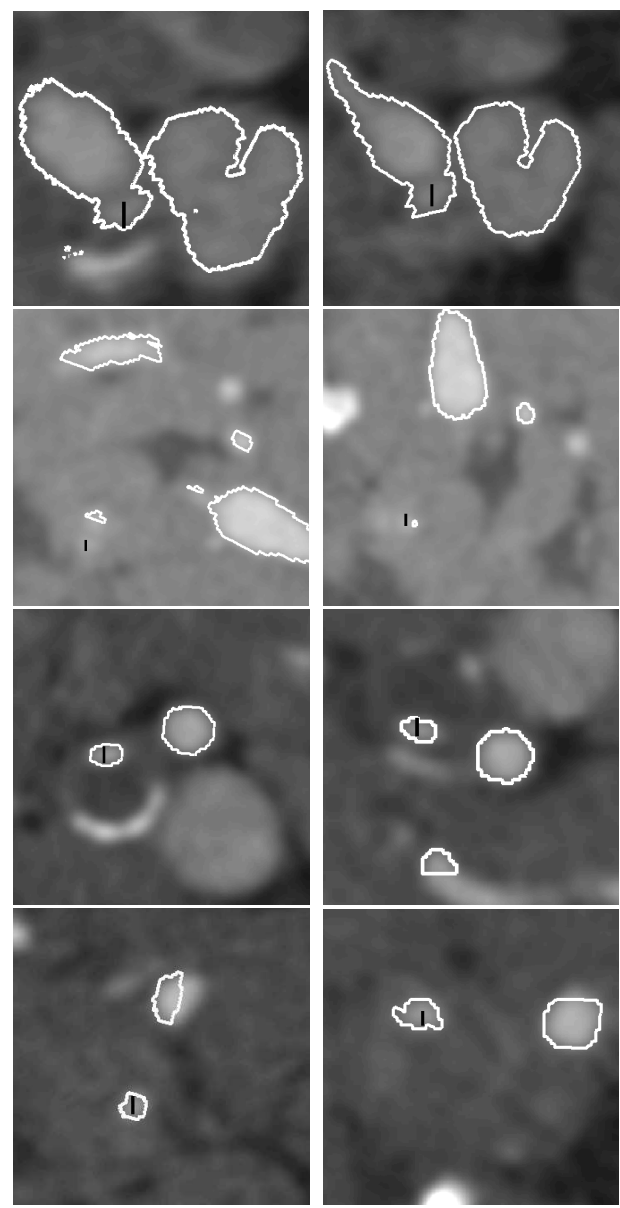

Fig. 8. For ICAs (g) to (j) we show for some measurements the corresponding MPRs at the location of the stenosis. We give in each row for a specific patient MPR views that were used by the observers for their evaluations. We tried to include from left to right at least two good results and two results that did not perform as well. In each picture we indicate the measurement of a radiologist (black line) and the segmentation of our method (white contours).

for ICA (a) and (h), some measurements of an observer were not comparable with the algorithm's result. In (a) the third MPR is oriented immediately after the bifurcation in a manner that the external and internal carotid artery are still not separated enough. The measurement of the observer in the last MPR did measure right next to the algorithm's result. Because of the same reasons for ICA $(\mathrm{h})$ the measurements in the last two MPRs could also not be evaluated. Furthermore, we could not measure sensible results for the ICA (g) because the observers selected MPR orientations for their measurements that were not suited for our evaluation approach. These planes were located immediately after the bifurcation of the carotid artery and oriented in such a manner that the internal and external carotid artery are still connected in the segmentations. In other words, the orientation is not exactly orthogonal to the centerline but a little bit oblique. 


\begin{tabular}{|c||c|c|c|c|c|}
\hline ICA & Mean Obs. & Mean Meth. & Mean Error & SD Obs. & SD Meth. \\
\hline a & 56,01 & 49,45 & $-6,56$ & 8,51 & 5,48 \\
b & 67,69 & 67,06 & $-0,63$ & 6,76 & 3,74 \\
c & 54,25 & 70,79 & 16,54 & 7,90 & 7,93 \\
d & 66,65 & 54,38 & $-12,27$ & 8,47 & 16,02 \\
e & 66,59 & 55,97 & $-10,62$ & 3,77 & 5,27 \\
f & 39,50 & 48,22 & 8,72 & 9,74 & 9,86 \\
g & & & & & \\
h & 63,83 & 72,41 & 8,58 & 3,12 & 10,90 \\
i & 65,22 & 65,42 & 0,20 & 3,83 & 4,69 \\
j & 66,08 & 60,13 & $-5,94$ & 5,38 & 1,18 \\
\hline
\end{tabular}

Table 2

The mean degree of stenosis and its standard deviation (SD) is shown for each ICA both according to the observers and according to the method.

Table 2 lists the obtained results in detail. We observed an average error of $7 \%$ compared to the manual measurements of the observers. For each ICA, the mean degree of stenosis according to the observers and the mean degree of stenosis according to the method based upon each observer is calculated. We also give the standard deviation (SD) of the observers and of the method.

\section{Discussion and Conclusion}

We introduced a semi-automatic method dealing with vessel segmentation and quantification in contrast-enhanced CTA data sets of stenosed internal carotid arteries. Calcified or plaque-attached arterial walls have similar intensity values as the arterial lumen, especially in their border regions. Therefore, the most challenging task for the presented algorithm was to successfully segment only the arterial lumen, while excluding all kinds of plaques from the segmented regions. Today, the degree of stenosis is not automatically quantifiable by means of software tools and in the clinical environment the doctor has to employ the evaluation manually. This approach considerably suffers from both intra- and inter-observer variability. In order to make it possible to segment the arterial lumen only, we presented a new level-set based method with only minimal user interaction (two clicks) that was inspired by the ideas of the two-phase level-set method of Chan and Vese.

We evaluated the method with four phantoms and with ten CTA data sets 
of different patients. Our method had estimated rather accurately the degree of stenosis for phantoms (a) and (b), whereas the degree of stenosis had been overestimated for phantoms (c) and (d). It is shown in Table 1 that the error increases when examining vessels with small diameters. Thus, the error between reality and the result of our method is inversely proportional to the minimal diameter of the stenosis. This comes from the applied regularization based on mean curvature, thus vessels with a rather small diameter become more regularized. As the error can increase to nearly $10 \%$, this is definitely a shortcoming of the presented method, which needs to be addressed in future research.

In the experiments with real data sets (see section 4.3.2) the results were many times in excellent agreement with that of the observers. Compared to the observers the error made by the method was only in the subpixel range for $75.5 \%$ of all measurements. The standard deviations attest our method superior results for the ICAs (a), (b) and (j). For the ICAs (d) and (h) our method obeys a significantly higher SD, both ICAs had lots of calcified plaques. For the other ICAs, the performance of the method and of the observers were comparable. The ICAs (c), (e), (i) and (j) were satisfactorily segmented. They had only non-calcified plaques at the part of stenosis. However, for the other ICAs few border regions of calcified plaques were still included. In the data sets (a) and (g) the internal jagular vein, which is located in close contact to the carotid artery, is also contrast enhanced. Our method included parts of this veinous structure within the segmentation. We observed that it is dependent on the estimate points whether the vein is included within the segmentation. The intensity of the vein voxels is slightly lower than that of lumen voxels. Therefore, the method should be improved to completely avoid the segmentation of vein voxels to ensure no influence on the quantification result.

Because no deterministic measurements were possible by the manual evaluation procedure of the observers, it was not easy to evaluate the performance of the method. The obtained results attested the method good segmentation results when compared to the measurements of the observers. However, it has to be mentioned that a detailed analysis of the performance of the method would need an automated quantification procedure that is independent of the different observers. Currently, the results depend not only on the MPR view of measurement but also on the selection of points from each observer. For that reason, we plan to develop a quantification procedure that measures the minimal diameter of arterial lumen in planes perpendicular to the centerline of the vessel in-between the already defined two starting points. Thereby we will be able to compute the degree of stenosis automatically within the resulting profile. Then fully reliable and deterministic quantifications should be achievable with our method.

In order to further improve the segmentation results, more influence from the 
mean-curvature regularization would be desirable. During the evaluation we noticed that there is a high potential for regularizing the segmentation especially in border regions of calcified plaques, which are mostly located at the area of the stenosis. But we were not able to use this regularizing potential because it tended to disconnect the segmentation in the stenosed parts. The topology of all kinds of vessels can be compared with a tubular structure characterized by high mean-curvatures at their surfaces. Since for vessel structures the curvature in the plane perpendicular to the vessel direction is always large, only the curvature in the longitudinal direction of the vessel should be used for regularization. In an ongoing project we try to significantly improve the segmentations especially in the stenosed parts of high intensity calcified plaques by ignoring the mean curvature perpendicular to the vessel axis.

In conclusion, it had been a very challenging task to segment only the arterial lumen of vascular structures in CTA. The obtained results look promising enough to assume that the method will be able to compute superior results compared to the observers regarding accuracy and reproducibility. With further improvements we believe that the method could be used as a tool for achieving deterministic analysis in the area of stenosis quantification within CTA data sets.

\section{Acknowledgments}

We would like to thank OA. PD. Dr. med. Ulrich Baum and Dr. med. Katharina Anders for scoring the clinical data. We would also like to thank Andreas Wimmer for the implementation of the geodesic active contour model. HipGraphics Inc. provided the clinical software "InSpace3D".

\section{References}

[1] G. Herold. Innere Medizin. G. Herold, Köln, 2004.

[2] European Carotid Surgery Trialists' (ECST) Collaborative Group. MRC European carotid surgery trial: Interim results for symptomatic patients with severe (70-90\%) or with mild (0-29\%) carotid stenosis. Lancet, 337:1235-1243, 1991.

[3] G.J. Hankey, C.P. Warlow, and A.J. Molyneux. Complications of cerebral angiography for patients with mild carotid territory ischaemia being considered for carotid endarterectomy. Journal of Neurology, Neurosurgery and Psychatry, 53(7):542-548, 1990. 
[4] C.P. Derdeyn, W.J. Powers, and C.J. Moran. Role of Doppler US in screening for carotid atherosclerotic disease. Radiology, 197(3):635-643, 1995.

[5] M. Nonent, J.M. Serfaty, and N. Nighoghossian. Concordance rate differences of 3 noninvasive imaging techniques to measure carotid stenosis in clinical routine practise. Stroke, 35(3):682-686, 2004.

[6] S. Osher and J.A. Sethian. Fronts propagating with curvature-dependent speed: Algorithms based on Hamilton-Jacobi formulations. Journal of Computational Physics, 79:12-79, 1988.

[7] T.F. Chan and L.A. Vese. Active contours without edges. IEEE Transactions on Image Processing, 10(2):266-277, February 2001.

[8] A. Kanitsar, D. Fleischmann, R. Wegenkittl, D. Sandner, P. Felkel, and Eduard Gröller. Computed tomography angiography: a case study of peripheral vessel investigation. In Proceedings of the conference on Visualization '01, pages 477480. IEEE Computer Society, 2001.

[9] I. Isgum and B. van Ginneken. CT segmentation programs extract calcifications. Diagnostic Imaging Europe, 19:11-16, 2003.

[10] I. Isgum, B. van Ginneken, and M.A. Viergever. Automatic detection of calcifications in the aorta from abdominal CT scans. Computer Assisted Radiology and Surgery, pages 1037-1042, 2003.

[11] I. Isgum, B. van Ginneken, and M. Olree. Automatic detection of calcifications in the aorta from CT scans of the abdomen. Academic Radiology, 11:247-257, 2004 .

[12] I. Isgum, B. van Ginneken, and M. Prokop. A pattern recognition approach to automated coronary calcium scoring. volume 3 of International Conference on Pattern Recognition, pages 746-749, 2004.

[13] M. van Straten, H.W. Venema, G.J. Streekstra, J.A. Reekers, G.J. den Heeten, and C.A. Grimbergen. Removal of arterial wall calcifications in CT angiography by local subtraction. Medical Physics, 30(5):761-770, 2003.

[14] R. Manniesing, B.K. Velthuis, M.S. van Leeuwen, I.C. van der Schaaf, P.J. van Laar, and W.J. Niessen. Level set based cerebral vasculature segmentation and diameter quantification in CT angiography. Medical Image Analysis, 10(2):200$214,2006$.

[15] A.F. Frangi and W.J. Niessen. Quantitative analysis of vascular morphology from 3d mr angiograms: In vitro and in vivo results. Magnetic Resonance in Medicine, 45(2):311-322, 2001.

[16] C.M. van Bemmel and W.J. Niessen. Semiautomatic segmentation and quantification of the internal carotid artery from 3D contrast-enhanced MR angiograms. Magnetic Resonance in Medicine, 51(4):753-760, 2004.

[17] A.F. Frangi, W.J. Niessen, K.L. Vincken, and M.A. Viergever. Multiscale vessel enhancement filtering. volume 1496 of Lecture Notes in Computer Science, pages 130-137. Springer Verlag, Berlin, 1998. 
[18] L.M. Lorigo, O.D. Faugeras, and W.E.L. Grimson. Curves: Curve evolution for vessel segmentation. Medical Image Analysis, 5(3):195-206, 2001.

[19] A. Yezzi and A. Tsai. Medical image segmentation via coupled curve evolution equations with global constraints. Technical report, University of California, Department of Mathematics, School of Electrical and Computer Engineering, Georgia Institute of Technology, 2000.

[20] T. Boskamp, D. Rinck, F. Link, B. Kuemmerlen, G. Stamm, and P. Mildenberger. A new vessel analysis tool for morphometric quantification and visualization of vessels in CT and MRI datasets. Radiographics, 24:287297,2004 .

[21] A. Gerhards, P. Raab, S. Herber, K.-F. Kreitner, T. Boskamp, and P. Mildenberger. Software-assisted CT-postprocessing of the carotid arteries. Fortschr Roentgenstr, 176:870-874, 2004.

[22] J. Weickert and G. Kühne. Fast methods for implicit active contour models. In N. Paragios S. Osher, editor, Geometric Level Set Methods in Imaging, Vision and Graphics, pages 43-58. Springer, New York, 2003.

[23] S. Osher and R. Fedkiw. Level Set Methods and Dynamic Implicit Surfaces, volume 153 of Applied Mathematical Sciences. Springer, New York, 2003.

[24] H. Scherl. Quantification and analysis of vessels in computer tomography data. Master's thesis, Institute of Pattern Recognition, Department of Computer Science, Friedrich-Alexander-Universität Erlangen-Nürnberg, 2004.

[25] K. Krissian and C.F. Westin. Fast and accurate redistancing for level set methods. In Roberto Moreno Diaz and Alexis Quesada Arencibia, editors, Computer Aided Systems Theory (EUROCAST'03), pages 48-51, Las Palmas de Gran Canaria, Spain, February 2003.

[26] D. Adalsteinsson and J.A. Sethian. A fast level set method for propagating interfaces. Journal of Computational Physics, 118(2):269-277, 1995.

[27] M. Lell, K. Anders, C. Leidecker, W. Lang, W. Bautz, and M. Uder. CTA of carotid artery with different scanner types. Radiologe, 44(10):967-974, 2004. 